\title{
A PARTITION RESULT WHICH IMPLIES FINE'S IDENTITY
}

\author{
WEI-YUEH CHEN and SEN-SHAN HUANG* \\ Department of Mathematics, National Changhua University of Education, Changhua City 50058, Taiwan \\ e-mail: shuang@math.ncue.edu.tw
}

(Received 10 December, 2004; accepted 11 March, 2005)

IN MEMORY OF THE LATE PROFESSOR ROBERT A. RANKIN

\begin{abstract}
In this article, we derive a partition result and employ its special case to offer a new proof for an identity of N. J. Fine.
\end{abstract}

2000 Mathematics Subject Classification. Primary 11B65; Secondary 05A19.

1. Introduction. Euler was the first mathematician who laid the foundations of the theory of partitions and proved many beautiful partition theorems [12]. One significant example is that

the number of partitions of a positive integer $n$ into distinct parts is equal to the number of partitions of $n$ into odd parts.

Moreover, Euler's approach in [12] of the utilization of generating functions provided a powerful analytic method for future developments in the theory of partitions since then. For example, the result quoted above is equivalent to the identity

$$
\prod_{n=1}^{\infty}\left(1+q^{n}\right)=\prod_{n=1}^{\infty} \frac{1}{1-q^{2 n-1}} .
$$

Here and in the sequel, we always assume that $|q|<1$.

Fine [13] [14, pp. 46-47] established two refinements of Euler's theorem above. One of the two refinements states that

the number of partitions of $n$ into distinct parts with largest part $k$ equals the number of partitions of $n$ into odd parts such that $2 k+1$ equals the largest part plus twice the number of parts,

which, in the language of generating functions, says that

$$
\sum_{j=0}^{\infty}(-q ; q)_{j} q^{j+1} t^{j+1}=\sum_{m=0}^{\infty} \frac{t^{m+1} q^{2 m+1}}{\left(t q ; q^{2}\right)_{m+1}},
$$

where we adopt the standard notation $(a ; q)_{0}=1,(a ; q)_{n}=(1-a) \cdots\left(1-a q^{n-1}\right)$, for $n \in \mathbb{N}$, and $(a ; q)_{\infty}=\lim _{n \rightarrow \infty}(a ; q)_{n}$.

\footnotetext{
*Corresponding author.
} 
As for (1.2), refer to Andrews' book [5, p. 27] where he gave a neat (but technical) proof by repeated use of $q$-analog of the binomial theorem and Heine's fundamental transformation. In [1], Andrews offered two proofs for Fine's theorem. One follows directly from a basic hypergeometric series identity of Andrews. The other is a combinatorial approach in which Andrews modified Sylvester's graph theoretic proof of Euler's theorem to prove Fine's result.

In the sequel, we will refer to the identity (1.2) as Fine's identity. Note that, when $t=1$, the identity (1.2) reduces to

$$
\sum_{j=0}^{\infty}(-q ; q)_{j} q^{j+1}=\sum_{m=0}^{\infty} \frac{q^{2 m+1}}{\left(q ; q^{2}\right)_{m+1}} .
$$

which implies Euler's identity (1.1) by appealing to the equality that

$$
\prod_{n=1}^{\infty}\left(1+f_{n}(q)\right)=1+\sum_{n=1}^{\infty}\left(\prod_{j=1}^{n-1}\left(1+f_{j}(q)\right)\right) f_{n}(q),
$$

with $f_{j}(q)$ chosen to be $q^{j}$ and $q^{2 j-1} /\left(1-q^{2 j-1}\right)$, respectively, for our purpose.

In Section 2, we will establish a partition theorem (Theorem 2.1) which not only is an interesting result on its own but also leads to a new proof of (1.2). Indeed, the key idea in the proof of Theorem 2.1 comes from an elementary result of the theory of partitions. On the other hand, Theorem 2.1 can be transformed into a $q$-series identity through the utilization of generation functions. This resulting identity is stated in Theorem 2.3 in which we would like to draw your attention to the symmetry between $2 k+1$ and $2 l$.

In Section 3, we employ a special case of Theorem 2.3, namely Corollary 2.4, to derive a functional equation for the function on the right side of (1.2). This functional equation enables us to establish Fine's identity. Finally, in Section 4, we give an analytic proof of Theorem 2.3 by appealing to a series-product identity of Cauchy.

\section{The main results.}

TheOREM 2.1. Let $M, N, k, l$ be positive integers. Let $P_{1}(M, N ; k, l)$ denote the number of partitions of $M$ into $N$ parts in which all the parts are either $2 k+1$ or positive even integers belonging to the set $\{2 l, 2 l+2, \ldots, 2 l+(2 m-2)\}$. (Here $m$ is the number of repetitions of $2 k+1$ as a part.) Let $P_{2}(M, N ; k, l)$ denote the number of partitions of $M-1-2(k-l)$ into $N$ parts in which all the parts are either $2 l$ or positive odd integers belonging to the set $\{2 k+1,2 k+3, \ldots, 2 k+(2 n-1)\}$. (Here $n$ is the number of repetitions of $2 \mathrm{l}$ as a part.) Then

$$
P_{1}(M, N ; k, l)=P_{2}(M, N ; k, l) .
$$

We should emphasize that the values of $m$ and $n$ in Theorem 2.1 depend on the partitions under consideration. The special case $k=l=1$ in Theorem 2.1 yields the following interesting result.

Corollary 2.2. The number of partitions of $M$ into $N$ parts in which all the parts are either 3 or positive even integers belonging to the set $\{2,4, \ldots, 2 m\}$ (where $m$ is the 
number of repetitions of 3 as a part) is equal to the number of partitions of $M-1$ into $N$ parts in which all the parts are either 2 or positive odd integers belonging to the set $\{3,5, \ldots, 2 n+1\}$. (Here $n$ is the number of repetitions of 2 as a part.)

Proof of Theorem 2.1. Define $\mathbb{P}_{1}$ and $\mathbb{P}_{2}$ to be the set of all the partitions counted in $P_{1}(M, N ; k, l)$ and $P_{2}(M, N ; k, l)$, respectively. For convenience, we use the notation

$$
\left\langle(2 k+1)^{m},(2 l)^{j_{1}},(2 l+2)^{j_{2}}, \ldots,(2 l+2 m-2)^{j_{m}}\right\rangle
$$

to denote a typical partition in $\mathbb{P}_{1}$, where the nonnegative exponents $m, j_{1}, \ldots$, and $j_{m}$ indicate the numbers of repetitions of the parts $2 k+1,2 l, \ldots$, and $2 l+2 m-2$, respectively. Similar notation will be used for partitions in $\mathbb{P}_{2}$. Next, we define a mapping $\Phi$ from $\mathbb{P}_{1}$ to $\mathbb{P}_{2}$ by

$$
\left\langle(2 k+1)^{m},(2 l)^{j_{1}}, \ldots,(2 l+2 m-2)^{j_{m}}\right\rangle \mapsto\left\langle(2 l)^{n},(2 k+1)^{h_{1}}, \ldots,(2 k+2 n-1)^{h_{n}}\right\rangle,
$$

where $n=N-(m-1), \sum_{i=1}^{m} j_{i}=n-1, \sum_{i=1}^{n} h_{i}=m-1$, and $\left\langle 1^{h_{2}}, \ldots,(n-1)^{h_{n}}\right\rangle$ is the conjugate partition of $\left\langle 1^{j_{2}}, \ldots,(m-1)^{j_{m}}\right\rangle$. Next, by appealing to the fact that

the number of partitions of $c$ in which there are at most $n-1$ parts and all the parts are not greater than $m-1$ is equal to the number of partitions of $c$ in which there are at most $m-1$ parts and all the parts are not greater than $n-1$,

it is evident that $\Phi$ is indeed a well-defined function from $\mathbb{P}_{1}$ to $\mathbb{P}_{2}$ and is invertible from $\mathbb{P}_{1}$ to $\mathbb{P}_{2}$. Hence, $\Phi$ is a bijection and so $\left|\mathbb{P}_{1}\right|=\left|\mathbb{P}_{2}\right|$. This proves the theorem.

REMARK 1 . Indeed, our proof of Theorem 2.1 is equivalent to a very nice graphic theoretic proof by employing so called 2-modular partitions [5]. We sketch the proof by the following diagrams.

For the partition (2.1) in $\mathbb{P}_{1}$, we first extract $2 l$ from each even part, apply 2modular partition process to place the resulting even parts column by column in the table

\begin{tabular}{rrrrr|l}
$2 l$ & $2 l$ & $2 l$ & $\ldots$ & $2 l$ & \\
\hline 2 & 2 & 2 & $\ldots$ & & \\
2 & 2 & 2 & $\ldots$ & \\
$\vdots$ & $\vdots$ & $\vdots$ & $\ddots$ & & \\
& & & & & \\
\hline & & & &
\end{tabular}

and then add the odd parts $2 k+1$ ( $m$ of them) on the left side of the table. So we have

\begin{tabular}{l|rrrrr|l} 
& $2 l$ & $2 l$ & $2 l$ & $\ldots$ & $2 l$ & \\
\hline $2 k+1$ & 2 & 2 & 2 & $\ldots$ & & \\
$2 k+1$ & 2 & 2 & 2 & $\ldots$ & & \\
$\vdots$ & $\vdots$ & $\vdots$ & $\vdots$ & $\ddots$ & & \\
$2 k+1$ & & & & & \\
\hline $2 k+1$ & & & & &
\end{tabular}


Next, we delete the $2 k+1$ at the bottom and add a $2 l$ at the end of the first row so that the table becomes

\begin{tabular}{l|rrrrr|r} 
& $2 l$ & $2 l$ & $2 l$ & $\ldots$ & $2 l$ & $2 l$ \\
\hline $2 k+1$ & 2 & 2 & 2 & $\ldots$ & & \\
$2 k+1$ & 2 & 2 & 2 & $\ldots$ & & \\
$\vdots$ & $\vdots$ & $\vdots$ & $\vdots$ & $\ddots$ & & \\
$2 k+1$ & & & & & \\
\hline & & & & &
\end{tabular}

Finally, reading each $2 l$ in the first row as a part and the other rows in rows gives a partition in $\mathbb{P}_{2}$. On the other hand, the process above is evidently reversible and so the proof is completed.

By using the terminology of generating functions, we can transform Theorem 2.1 into an identity.

THEOREM 2.3. For $|q|<1$ and positive integers $k$ and $l$, we have

$$
q^{2 l} \sum_{m=1}^{\infty} \frac{t^{m} q^{(2 k+1) m}}{\left(t q^{2 l} ; q^{2}\right)_{m}}=q^{2 k+1} \sum_{m=1}^{\infty} \frac{t^{m} q^{2 l m}}{\left(t q^{(2 k+1)} ; q^{2}\right)_{m}}
$$

Notice that the beauty of Theorem 2.3 lies on the symmetry between $2 l$ and $2 k+1$. By taking $k=l=1$ in Theorem 2.3 or by Corollary 2.2 , we have

Corollary 2.4 .

$$
\sum_{m=1}^{\infty} \frac{t^{m} q^{3 m}}{\left(t q^{2} ; q^{2}\right)_{m}}=q \sum_{m=1}^{\infty} \frac{t^{m} q^{2 m}}{\left(t q^{3} ; q^{2}\right)_{m}}
$$

In next section, we will employ Corollary 2.4 to derive a functional equation which enables us to establish Fine's identity.

3. A new proof of Fine's identity. For convenience, define

$$
G(t)=\sum_{m=0}^{\infty} \frac{t^{m+1} q^{2 m+1}}{\left(t q ; q^{2}\right)_{m+1}}
$$


And then,

$$
\begin{aligned}
t q(1+G(t q)) & =t q\left(1+\sum_{m=0}^{\infty} \frac{t^{m+1} q^{3 m+2}}{\left(t q^{2} ; q^{2}\right)_{m+1}}\right)=t q\left(1+\sum_{m=1}^{\infty} \frac{t^{m} q^{3 m-1}}{\left(t q^{2} ; q^{2}\right)_{m}}\right) \\
& =t q\left(1+\sum_{m=1}^{\infty} \frac{t^{m} q^{2 m}}{\left(t q^{3} ; q^{2}\right)_{m}}\right) \quad(\text { by Corollary } 2.4) \\
& =t q \sum_{m=0}^{\infty} \frac{t^{m} q^{2 m}}{\left(t q^{3} ; q^{2}\right)_{m}}=(1-t q) \sum_{m=0}^{\infty} \frac{t^{m+1} q^{2 m+1}}{\left(t q ; q^{2}\right)_{m+1}} \\
& =(1-t q) G(t) .
\end{aligned}
$$

Hence, we arrive at the functional equation

$$
G(t)=t q(1+G(t)+G(t q)) .
$$

Write

$$
G(t)=\sum_{j=1}^{\infty} A_{j} t^{j}
$$

where $A_{j}$ is a polynomial of $q$. Observe that $A_{1}=q$. This can be seen by rewriting $G(t)$ as

$$
G(t)=\frac{t q}{(1-t q)}+\sum_{m=1}^{\infty} \frac{t^{m+1} q^{2 m+1}}{\left(t q ; q^{2}\right)_{m+1}} .
$$

By (3.1), we have

$$
\sum_{j=1}^{\infty} A_{j} t^{j}=t q\left(1+\sum_{j=1}^{\infty} A_{j} t^{j}+\sum_{j=1}^{\infty} A_{j} q^{j} t^{j}\right) .
$$

For $N>1$, by equating the coefficients of $t^{N}$ on both sides of the last equality, we obtain

$$
\begin{aligned}
A_{N} & =q\left(A_{N-1}+q^{N-1} A_{N-1}\right) \\
& =q\left(1+q^{N-1}\right) \cdot A_{N-1} \\
& =q\left(1+q^{N-1}\right) \cdots \cdot q(1+q) A_{1} \\
& =(-q ; q)_{N-1} q^{N} .
\end{aligned}
$$

Hence,

$$
G(t)=\sum_{j=1}^{\infty}(-q ; q)_{j-1} q^{j} t^{j}
$$

which is Fine's identity (1.2).

REMARK 2. The functional equation (3.1) for $G(t)$ can also be obtained by using results of N. J. Fine. More precisely, one can employ the equations (2.4) with $a=$ $-1, b=0$ and $[\mathbf{1 4},(20.72)]$ to derive (3.1). We leave the details to the reader. 
In addition to Fine's theorem, various generalizations of Euler's theorem are known. A famous refinement of Euler's theorem is due to Sylvester [18] which says that the number of partitions of $n$ into odd parts with exactly $k$ distinct parts appearing equals the number of partitions of $n$ into distinct parts such that exactly $k$ sequences of consecutive integers occur in each partitions. (See [5, Theorem 2.12], [3], [17].) Bessenrodt [7] obtained a generalization for Fine's theorem while constructing a bijection for Lebesgue's partition identity. (Also, see [16].) Bousquet-Mélou and Eriksson [8] introduced a new concept named lecture hall partitions and proved the so-called Lecture Hall Theorem whose limiting case yields Euler's identity (1.1). For references to lecture hall partitions, we refer the reader to [4], [6], [9], [10] and [19]. Recently, the authors and S.-D. Chen [15] established an octuple product identity from which Euler's identity (1.1) follows as a special case.

4. An analytic proof of Theorem 2.3. In the final section, we offer an analytic proof of Theorem 2.3 with the help of the following result which was originally due to Cauchy.

LeMMA 4.1. [11, p. 46] For any positive integer $m$,

$$
\frac{1}{(z ; q)_{N}}=\sum_{j=0}^{\infty}\left[\begin{array}{c}
N+j-1 \\
j
\end{array}\right]_{q} z^{j},
$$

where $\left[\begin{array}{c}n \\ m\end{array}\right]_{q}$ is the Gaussian polynomial.

Now, by Lemma 4.1 and the fundamental property of the Gaussian polynomial, namely, $\left[\begin{array}{l}n \\ m\end{array}\right]_{q}=\left[\begin{array}{c}n \\ n-m\end{array}\right]_{q}$, we have

$$
\begin{aligned}
& q^{2 l} \sum_{m=1}^{\infty} \frac{t^{m} q^{(2 k+1) m}}{\left(t q^{2 l} ; q^{2}\right)_{m}} \\
& =q^{2 l} \sum_{m=1}^{\infty} t^{m} q^{(2 k+1) m} \sum_{n=0}^{\infty}\left[\begin{array}{c}
m+n-1 \\
n
\end{array}\right]_{q^{2}}\left(t q^{2 l}\right)^{n} \\
& =\sum_{n=0}^{\infty} \sum_{m=1}^{\infty}\left[\begin{array}{c}
m+n-1 \\
n
\end{array}\right]_{q^{2}} t^{m+n} q^{2 k m+m+2 n l+2 l} \\
& =\sum_{n=1}^{\infty} \sum_{m=0}^{\infty}\left[\begin{array}{c}
m+n-1 \\
n-1
\end{array}\right]_{q^{2}} t^{(m+1)+(n-1)} q^{2 k(m+1)+(m+1)+2(n-1) l+2 l}
\end{aligned}
$$

(Here, we replace $n$ and $m$ by $n-1$ and $m+1$, respectively.)

$$
\begin{aligned}
& =q^{2 k+1} \sum_{n=1}^{\infty} \sum_{m=0}^{\infty}\left[\begin{array}{c}
m+n-1 \\
m
\end{array}\right]_{q^{2}} t^{m+n} q^{2 k m+m+2 n l} \\
& =q^{2 k+1} \sum_{n=1}^{\infty} t^{n} q^{2 n l} \sum_{m=0}^{\infty}\left[\begin{array}{c}
m+n-1 \\
m
\end{array}\right]_{q^{2}} t^{m} q^{(2 k+1) m} \\
& =q^{2 k+1} \sum_{n=1}^{\infty} \frac{t^{n} q^{2 n l}}{\left(t q^{2 k+1} ; q^{2}\right)_{n}} .
\end{aligned}
$$

This completes the proof of Theorem 2.3. 
Acknowledgements. We would like to thank Professor B. C. Berndt for his invaluable suggestions during the preparation of this article.

\section{REFERENCES}

1. G. E. Andrews, On basic hypergeometric series, mock theta functions, and partitions. II., Quart. J. Math. Oxford Ser. (2) 17 (1966), 132-143.

2. G. E. Andrews, $q$-Identities of Auluck, Carlitz, and Rogers, Duke Math. J. 33 (1966), $575-582$.

3. G. E. Andrews, On generalizations of Euler's partition theorem, Michigan Math. J. 13 (1966), 491-498.

4. G. E. Andrews, MacMahon's partition analysis. I., The lecture hall partition theorem in Mathematical essays in honor of Gian-Carlo Rota (Cambridge, MA, 1996), Progr. Math. 161 (1998), 1-22.

5. G. E. Andrews, The theory of partitions (Cambridge University Press, 1998).

6. G. E. Andrews, P. Paule, A. Riese and V. Strehl, MacMahon's partition analysis. V. Bijections, recursions, and magic squares, in Algebraic combinatorics and applications (Gö $\beta$ weinstein, 1999), (Springer-Verlag, 2001), 1-39.

7. C. Bessenrodt, A bijection for Lebesgue's partition identity in the spirit of Sylvester, Discrete Math. 132 (1994), 1-10.

8. M. Bousquet-Mélou and K. Eriksson, Lecture hall partitions, Ramanujan J. 1 (1997), $101-111$. $165-185$.

9. M. Bousquet-Mélou and K. Eriksson, Lecture hall partitions. II, Ramanujan J. 1 (1997),

10. M. Bousquet-Mélou and K. Eriksson, A refinement of lecture hall partitions, J. Combin. Theory Ser. A 86 (1999), 63-84.

11. A. Cauchy, Oeuvres, Ser. 1, Vol. 8, Gauthier-Villars, Paris.

12. L. Euler, Introductio in Analysin Infinitorum, Chapter 16, Marcum-Michaelem Bousquet, Lausannae.

13. N. J. Fine, Some new results on partitions, Proc. Nat. Acad. Sci. 34 (1948), 616-618.

14. N. J. Fine, Some basic hypergeometric series and applications, Mathematical Surveys and Monographs, 27 (Amer. Math. Soc., 1989).

15. S.-D. Chen, W.-Y. Chen and S.-S. Huang, A new construction of Ewell's octuple product identity, Indian J. Pure Appl. Math. 35 (2004), 1241-1253.

16. D. Kim and A. J. Yee, A note on partitions into distinct parts and odd parts, Ramanujan J. 3 (1999), 227-231.

17. V. Ramamani and K. Venkatachaliengar, On a partition theorem of Sylvester, Michigan Math. J., 19 (1972), 137-140.

18. J. J. Sylvester, A constructive theory of partitions arranged in three acts, an interact, and an exodion, Amer. J. Math. 5, 251-330; 6, 334-336 (or pp. 1-83 of the Collected Papers of J. J. Sylvester, Vol. 4, Cambridge University Press, 1912; reprinted by Chelsea, New York, 1974). 262.

19. A. J. Yee, On the combinatorics of lecture hall partitions, Ramanujan J. 5 (2001), 247 